\title{
Psychological Safety as Determinants of the Belief in Dangerous Online Communities Mediated by General and Online Social Anxiety
}

\author{
Jon-Chao Hong \\ Department of Industrial Education \\ National Taiwan Normal University \\ Taipei, Taiwan \\ tcdahong@gmail.com
}

\author{
Chin-Hao Hsu \\ Department of Industrial Education \\ National Taiwan Normal University \\ Taipei, Taiwan \\ henry780305@hotmail.com
}

\author{
Kai-Hsin Tai \\ Department of Industrial Education \\ National Taiwan Normal University \\ Taipei, Taiwan \\ star99xin@gmail.com
}

\begin{abstract}
This study explores the antecedents and outcomes of individual perceived safety in life. After factoring out beliefs and social anxiety, Facebook use was selected as a predictor for related psychological symptoms. A confirmatory factor analysis was applied to 230 email validated samples. Structural equation modeling was used to test the predictions for each construct. The results show that Psychological need of safety was positively correlated to both social anxieties and positively reflected to beliefs in dangerous online communities. The implication is that if the participants have high level of both social anxieties, then they have high levels of belief in dangerous online communities. The implication of these findings in conjunction with a number of discussions and recommendations for future research are provided.
\end{abstract}

Keywords-general social anxiety; online social anxiety; psychological needs for safety; belief in a dangerous online community

\section{INTRODUCTION}

People choose to gain precious knowledge and endeavor positive interactive connections with others via the internet. However, others use the internet to harm innocent people and/ or commit illegal acts. According to the survey, Taiwanese citizens' average belief in online security is not high. Based on the "2007 Taiwan Internet Security Survey" from the ALS/NII Production Development Association, only $34 \%$ of the population hold positive beliefs toward internet security, which is lower than the levels of developed countries in Europe and North America (US 53\%; Germany $43 \%$ ). This phenomenon underplayed the belief in dangerous online communities and lead users to hesitate the presentation of information to friends on virtual worlds. Existing research has shown that behavior and belief in participation vary by type of online community [1] and pertain to anxiety-related dispositions elevated. Studies report significant associations between personality traits and aspects of social network use. The belief of the social world has a variety of evidence consistent with the basic premise of social anxiety to show that perceptions of the social world that are dangerous should be predicted by individual needs for safety reasons.

Facebook was chosen as the virtual environment to study, because most other alternatives were constrained by limited functionality. In Facebook, a great number of different activities are available and users have tools to promote oneself to establish new connections with others. Therefore, the aim of the current study was to look at Facebook users' psychological needs in a manner to understand user psychological anxiety and beliefs in dangerous online communities that underlie their use of the virtual world. This understanding is an important basis for researchers that work on virtual worlds.

\section{LITERATURE REVIEW}

Oldmeadow, Quinn, and Kowert (in press) stated that "with the growth in popularity of social networking sites (SNSs) such as Facebook, Myspace and Twitter, new forms of social interaction have emerged that differ in important ways from the offline interactions more typically studied by social psychologists". These studies indicated that the believability of the medium influenced how users will view the credibility of the information offered and may react negatively towards the social network [2]. Accordingly, this study takes Facebook as a research sample to understand user conditions. From a psychological need for safety points of view, we investigate user social network anxiety and its influence on real social life problems as well as belief in dangerous online communities.

\section{RESEARCH HYPOTHESES}

The present study aimed to examine the correlation between psychological safety, general social anxiety, online social anxiety, and a belief in a dangerous online community. Accordingly, the hypotheses of this study were:

H1: General social anxiety is significantly correlated to a belief in a dangerous online community. 
$\mathrm{H}$ 2: Online social anxiety is significantly correlated to a belief in a dangerous online community.

H3: General social anxiety is significantly correlated to online social anxiety

H4: Psychological safety is significantly correlated to general social anxiety.

H5: Psychological safety is significantly correlated to online social anxiety.

\section{RESEARCH DESIGN}

\section{A. Research participants}

There were 505 questionnaires returned. After deletion of 275 ineffective questionnaires, there were 230 effective questionnaires collected. We achieved an effective questionnaire return rate of $46 \%$.

\section{B. Research instruments}

The survey instruments contained psychological safety, general and online social anxiety, and a belief in a dangerous online community scale that would be described in the following paragraph

Psychological safety: Maslow [3] referred to psychological safety, questions that dealt with safety and security that ask for the behavioral consequence of risk factors, such as criticism, put-downs, and name-calling, may be overlooked or accepted by the participant that is constitutive of a psychological context of safety. Participants rate on a five-point scale the extent to which they feel each statement is characteristic or true for them.

General and online social anxiety: The two social anxiety measures adapted from Mattick and Clarke's [4] Social Interaction Anxiety Scale, which taps anxiety in interpersonal and social situations.

Belief in a dangerous online community: The scale adapted from Altemeyer's [5] "Belief in a Dangerous World scale", measures an examination of chronic beliefs about interpersonal danger. Scoring high on this measure indicates greater beliefs that the world is a dangerous place where one must protect oneself from interpersonal harm.

\section{DATA ANALYSIS}

Based on the research evidence, we used SPSS 20 software to perform reliability analysis to determine the reliability of the questionnaires, and an item analysis was used to examine the validity and correlation of the Scales. The study also used Amos 20 to examine the structural equation modeling (SEM) of research hypotheses.

The results of these analyses showed that the internal consistency values for the scale were .840 to .869 , respectively, suggesting acceptable reliability for both values. We additionally examined convergent validity, which refers to the degree to which multiple items measure the same construct. Previous studies [6] suggested that evaluation can be made by checking if (1) the average variance of the extracted values was larger than .5 , and (2) the factor loadings of all items were significant and higher than .5 . Both of these conditions were met in the present study, indicating the acceptable convergent validity.

Items were excluded from a construct if the loading value was below $<.5$, because it signified the item was ambiguously loaded to a construct and was conceptually incoherent with other items on the construct [6], [7]. All these conditions were met to indicate acceptable convergent validity. Item analysis is also known as a discriminative ability of item analysis. The main purpose is to increase the validity of the questionnaire by delete non-discriminating items. All t-values of the constructs were significant (if t-value $>3.29$, representing $* * * p<.001)$, representing that all items were discriminative. All items to able to identify the degree of response of different samples, i.e., no items needed to be deleted.

\section{RESEARCH RESULTS}

The analysis was run in two steps. First step, the degree of linear relationship between each construct was calculated using the well-known Pearson's $r$ coefficient of correlation. Second step, we adopted Amos 20 for path modeling over covariance-based SEM.

\section{A. Correlation Analyses}

Table 1 shows that there were significant correlations between PS, GSA, OSA, and BDVC, i.e., they all belong to "moderate correlation". There is a certain degree of correlation among these continuous variables.

TABLE I. THE CORRELATION MATRIX

\begin{tabular}{ccccc}
\hline & PS & GSA & OSA & BDVC \\
\hline PS & 1 & & & \\
GSA & $.488 * * *$ & 1 & & \\
OSA & $.500 * * *$ & $.582 * * *$ & 1 & \\
BDVC & $.429 * * *$ & $.385 * * *$ & $.459 * * *$ & 1 \\
\hline
\end{tabular}

\section{B. Model goodness of fit test}

This study used SEM with Amos 20 to test the goodness of fit for the model. We adopted Hair's recommendations to set $\chi^{2} / \mathrm{df}<5$ as an acceptable level, together with multiple indicators to obtain a more objective conclusion to avoid power problems that arise using the Chi-square test in a large sample. The model was hypothesized as $\chi^{2}=657.200$, $\mathrm{df}=207 ; \chi^{2} / \mathrm{df}=2.183$, probability level $\mathrm{p}=.02<.05$, RMSEA $=.042$, GFI=.837, AGFI=.895, in which GFI and AGFI were more than .8 and RMSEA was lower than .05 , to represent that this model fit the data best.

Hair et al. [8] proposed that researchers should not only pay attention to the Chi-square values but should consider other fitness measures at the same time. The values of fitness were all larger than .9: $\mathrm{NFI}=.904$, RFI=.908, IFI=.928, TLI=.986, and CFI=.903. Overall, judging from the comprehensive indicators, the theoretical model fit the overall pattern of the data.

\section{Structural Equation Modeling (SEM)}

Figure 1 shows the results of the path relations among the hypotheses. We can see that all 5 Hypotheses were supported. Figure 2 indicates that the test of the antecedent of PS to the participant GSA and OSA was moderated supported, with standardized regression coefficients (SRC) 
of .176 and .246 ; the test of the antecedent of GSA to OSA was supported, with a SRC of .577; the test of the antecedent of GSA to BDVW was supported, with a SRC of .224; the test of the antecedent of OSA to BDVW was supported, with a SRC of .423. The hypothesis was proven to be significant and, therefore, was used to verify the theoretical model. The $\mathrm{R} 2$ value is the percentage of variation as explained by the exogenous variable to the endogenous variables, thus, representing the predictive ability of the research model. Path coefficients and R2 values indicated the fit of the structural models with the empirical data. Figure 1 shows the compilation of standardized path coefficients between constructs, test results, and explained variation (R2).

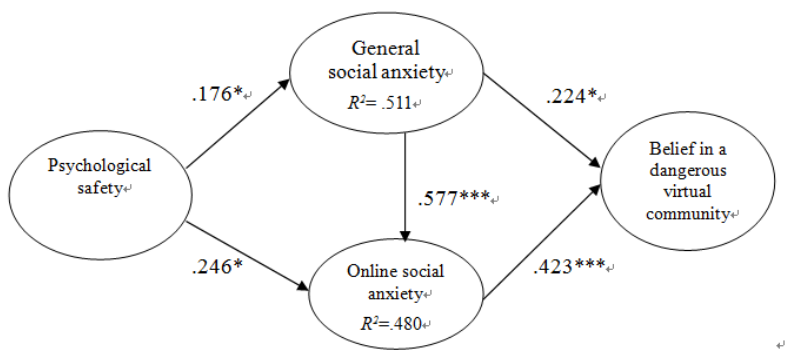

FIGURE I. RESULT OF RESEARCH MODEL

\section{FINDINGS}

The present study employed one of Maslow's psychological needs, the safety to examine the interrelatedness between general and online social anxiety and beliefs in a dangerous virtual world in relation to Facebook users. According to Maslow [3], psychological safety referred to a condition of feeling free or protected from the specific case of harm to the psyche, soul, or mind. The need for psychological safety is positively associated with both types of social anxiety and assured the belief in a dangerous virtual world.

The results of this study supported their assertions and found the high level of psychological safety the participants had, the high degree of general and online social anxiety. The results also supported by Caplan [9], which claimed the fact that individuals who lack security would often attempt to find their emotional reliance through social network, i.e., people differ substantially in terms of whether they believe the virtual world to be a dangerous environment, and generally vulnerable they feel to interpersonal sources of anxiety.

\section{REFERENCE}

11 T. Partala, "Psychological needs and virtual worlds: Case second life," International Journal of Human-Computer Studies, vol. 69, pp.787-800.

[2] N. B. Ellison, C. Steinfield, and C. Lampe, "The benefits of Facebook "Friends": Social capital and college students' use of online social network sites," Journal of Computer-Mediated Communication, vol. 12, pp. 1143-1168, 2007.

[3] A. H. Maslow, "Motivation and personality," New York: Harper and Row, 1970 .

[4] R. P. Mattick, and J. C. Clarke, "Development and validation of measures of social phobia, scrutiny fear and social interaction anxiety," Behavior Research and Therapy, vol 36, pp. 455-470, 1998.

[5] B. Altemeyer, "Enemies of freedom," San Francisco: Jossey-Bass, 1988.

[6] C. Fornell, and D. Larcker, "Structural equation models with unobservable variables and measurement error," Journal of Marketing Research, vol 18, pp. 39-50, 1981
[7] J. C. Nunnally, Psychometric theory (2nd ed.), New York: McGraw-Hill, 1978.

[8] J. Hair, B. Black, B. Babin, R.E. Anderson, and R.L. Tatham, Multivariate Data Analysis (7ed.), Englewood Cliffs, NJ: Prentice Hall, 2009.

[9] S. E. Caplan, "Relations among loneliness, social anxiety and problematic internet use," Cyber psychology \& Behavior, vol.10, pp. 234-242, 2007. 